\title{
CONGRESO MUNDIAL DE LA FEDERACIÓN INTERNACIONAL DE ASOCIACIONES DE PLACENTA, IFPA CHILE 2010
}

Desde el inicio de la vida humana (i.e. desde el momento de la concepción), el huevo fecundado requiere de un ambiente que lo proteja y le aporte los nutrientes, incluyendo el oxígeno, necesarios para su desarrollo. Las modificaciones sistémicas y locales, así como en la decidua materna, llevan finalmente a la implantación y la diferenciación del trofoblasto que luego dará origen a la placenta, son fenómenos claves para la gestación de un nuevo individuo.

La placenta es el órgano que protege, nutre y mantiene comunicado al feto con el ambiente extra uterino hasta el momento de su nacimiento. Estos fenómenos ocurren gracias a una compleja red funcional establecida entre la madre y el feto que regulan y favorecen el adecuado desarrollo y crecimiento, primero del embrión y luego del feto. Esta red funcional media el intercambio maternofetal de metabolitos y está constituido por una serie de tejidos: la sangre materna que desde las arterias uterinas llega a bañar las vellosidades placentarias, el sinciciotrofoblasto, el espacio estromal con células estromales (ej. fibroblastos y células con prolongaciones dendríticas y actividad macrofágica), la lámina de células endoteliales de la microcirculación vellositaria en el lado fetal de la placenta y la sangre fetal (1). Como producto de los fenómenos de transporte a través de las membranas plasmáticas de estos tejidos y del metabolismo extra e intra celular en estos tipos celulares surge el fenómeno de "señalización placentaria".

Este intrincado fenómeno fisiológico no solamente comprende el traspaso vectorial de metabolitos (ej. nutrientes, incluyendo el oxígeno, y productos de deshecho, incluyendo el dióxido de carbono, entre otros) desde la madre hacia el feto y desde el feto hacia la madre, para la nutrición y el bienestar del feto en desarrollo, sino también el traspaso de moléculas que regulan procesos fisiológicos cruciales para la vida fetal (ej., aminoácidos, agua, cito- quinas, nucleósidos, ácidos grasos, entre otros).

Este fenómeno también redunda en la regulación de procesos biológicos básicos en la madre lo cual altera funciones fisiológicas que le son determinantes, tales como la presión arterial en la preeclampsia $(2,3)$ o el nivel de D-glucosa plasmática en la diabetes gestacional $(4,5)$. Así, la disfunción de la placenta determina un estado fisiopatológico que perturba el buen estado de salud de la madre y el feto en desarrollo.

Chile, es un país en vías de desarrollo que presenta una alta incidencia de enfermedades asociadas con disfunción placentaria, tales como preeclampsia, diabetes gestacional y restricción del crecimiento intrauterino (6). Estas patologías se asocian con patologías en la madre y/o el feto y recién nacido, pero además se asocian con alteraciones metabólicas que llevan a "programar" o predisponer a los recién nacidos a la manifestación de otras patologías durante la vida adulta $(7,8)$, un fenómeno que podría, al menos en parte, ser también explicado o sostenido por modificaciones epigenéticas (9).

Con el fin de agrupar a investigadores multi e inter disciplinarios abocados a entender fenómenos involucrados en la función/disfunción placentaria se ha constituido la Federación Internacional de Asociaciones de Placenta, IFPA, (International Federation of Placenta Associations) (www.ifpa.com). IFPA se conforma por sus ramas Asociaciones de Investigación en Placenta de Australia y Nueva Zelandia (ANZPRA), Grupo Europeo de Placenta (EPG), Asociación de Placenta Japonesa (JPA) y las Asociaciones de Placenta de las Américas (PAA). Los investigadores que conforman estas agrupaciones se reúnen anualmente en forma alternada entre estos cuatro continentes. Por primera vez en su historia IFPA realizará el congreso mundial en América del Sur, particularmente en Chile en el mes de Septiembre del año 2010 (IFPA, Chile 
2010). Esta es sin duda una ocasión única para las agrupaciones latino-americanas de investigadores en placenta (LAPG), miembros de la PAA (reunidos ya en cuatro oportunidades en diferentes países en nuestra región, incluyendo Chile en el año 2005), para participar masivamente en Chile en el Congreso del año 2010, donde se privilegiará la discusión científica tanto clínica como básica en exposiciones plenarias, simposios, talleres de trabajo, comunicaciones y presentaciones en paneles. Los tópicos a cubrir contemplan todos los aspectos de la formación (implantación) y función de la placenta incluyendo desarrollo, histología, fisiología, metabolismo, endocrinología, microbiología, patología, inmunología, imagenología, diagnóstico, farmacología, biología celular, bioquímica y mecanismos moleculares asociados a la función/disfunción placentaria. Se considerará también estudios en aspectos clínicos acerca de la estructura y función de la placenta, así como estudios de implantación, placentación comparativa, neoplasia del trofoblasto y biología vascular de la unidad feto-placenta. Todos los estudios presentados a esta reunión serán publicados en la revista internacional Placenta (último factor de impacto = 3,238) (10).

Los tópicos a desarrollar en este congreso mundial de placenta incluyen desde lo general a mecanismos específicos que se conoce como causas, o que han sido propuestos como tales para la disfunción de la placenta en patologías del embarazo. Es vital comprender estos mecanismos en detalle para alcanzar un nivel de conocimiento en el cual las decisiones terapéuticas sean tomadas en base a los conocimientos que surgen de la investigación fundamental. Este prestigioso Congreso Mundial se presenta como una oportunidad para la interacción e integración de los conocimientos generados tanto en la práctica clínica de la obstetricia como aquellos generados por los investigadores fundamentales. En nombre de IFPA los instamos a participar en este Congreso, apoyando con ello el mejoramiento de la investigación en perinatología en Chile.

\section{Dr Luis Sobrevia L. PhD Dra Paola Casanello T. PhD.}

Laboratorio de Investigación en Perinatología, Departamento de Obstetricia y Ginecología, Facultad de Medicina, Pontificia Universidad Católica de Chile.

\section{REFERENCIAS}

1. Sobrevia L, Casanello P. Placenta function. En: Pérez Sánchez A, Donoso Siña E (eds). OBSTETRICIA. 6a ed. Santiago: Editorial Mediterráneo, 2009. (En prensa).

2. Myatt L. Placental adaptive responses and fetal programming. J Physiol 2006;572:25-30.

3. Escudero C, Sobrevia L. A hypothesis for preeclampsia: adenosine and inducible nitric oxide synthase in human placental microvascular endothelium. Placenta 2008;29:469-83.

4. Desoye G, Hauguel-de Mouzon S. The human placenta in gestational diabetes mellitus. The insulin and cytokine network. Diabetes Care 2007;30 Suppl 2: S120-6.

5. Jansson T, Myatt L, Powell TL. The role of trophoblast nutrient and ion transporters in the development of pregnancy complications and adult disease. Curr Vasc Pharmacol 2009. (En prensa).

6. Casanello P, Escudero C, Sobrevia L. Equilibrative nucleoside (ENTs) and cationic amino acid (CATs) transporters: implications in foetal endothelial dysfunction in human pregnancy diseases. Curr Vasc Pharmacol 2007;5:69-84.

7. Barker DJ, Osmond C. Infant mortality, childhood nutrition, and ischaemic heart disease in England and Wales. Lancet 1986;1:1077-81.

8. Gluckman PD, Hanson MA, Cooper C, Thornburg $\mathrm{KL}$. Effect of in utero and early-life conditions on adult health and disease. N Engl J Med 2008;359:61-73.

9. Krause B, Sobrevia L, Casanello P. Epigenetic: new concepts of old phenomena in vascular physiology. Curr Vasc Pharmacol 2009. (En prensa).

10. Journal Citations Reports 2008. Thompson Reuters, 2008. Hallado en: http://www.isiwebofknowledge.com 